\title{
Toward a Holistic Agricultural Student Recruitment Model: A National Analysis of the Factors Affecting Students' Decision to Pursue an Agricultural Related Degree
}

\author{
Antoine J. Alston ${ }^{\mathrm{a}}$, Richie Roberts ${ }^{\mathrm{b}}$, Chastity Warren English ${ }^{\mathrm{a}}$ \\ ${ }^{a}$ North Carolina Agricultural and Technical State University, ${ }^{b}$ Louisiana State University
}

\begin{abstract}
Currently, the agricultural industry struggles to fill positions with qualified agricultural workers. Therefore, it is critical to attract high caliber individuals to agricultural degree programs that are prepared to enter the workforce with the skills needed to navigate complex issues and problems. The purpose of this national study was to identify key factors that influence the recruitment of agriculture students at land-grant and non-landgrant universities. Using Chapman's model of student success as our conceptual lens, we tested 66 factors identified in the literature as successful recruitment strategies for colleges of agriculture based on students' personal characteristics as well as key external influences. We discovered statistically significant $(p<.05)$ differences existed based on students' gender and race/ethnicity. To better operationalize the findings from this study for U.S. colleges of agriculture, we developed the agricultural student recruitment model (ASRM). The model visually represents the distinct but intersecting factors that most profoundly influence students' academic degree decisions. Moving forward, we recommend colleges of agriculture use the ASRM as a tool to better resonate with populations that may lack representation in their degree programs and the state's agricultural industry.
\end{abstract}

Keywords: agriculture, student, recruitment, degree, decision

\section{Introduction}

Our global population is projected to exceed nine billion by 2050; therefore, we face unprecedented challenges to produce sufficient food, feed, fiber, and biofuel feedstock. Strengthening the agricultural industry is essential to meeting this challenge (Food and Agriculture Organization of The United Nations, 2017). To fulfill such demands, agriculturalists will be required to produce more food over the next 40 years than has been produced over the past 10,000 years combined. Further, agriculturalists will be required to produce sufficient biofuel feedstock to ensure a secure and independent energy supply on a land area with fewer inputs (United Nations, 2015). Failure to meet these goals could cause food insecurity in many parts of the world, leading to instability in the global, 
geopolitical landscape. Coupled with this challenge is the growing demand for sustainable agricultural practices and locally grown food. As a result, agricultural producers will also be required to provide fresh food products for changing dietary and health habits of individuals across the globe. The United Nation's Food and Agricultural Organization (2015) estimated that agriculture collectively accounts for $40 \%$ of the current global workforce. However, to meet the aforementioned demands, more skilled leaders and laborers must be recruited into the agricultural sector, which will have to be trained through the nation's land grant and non-land grant colleges of agriculture. With this in mind, these institutions will have to identify mechanisms and strategies to attract potential students into the academic programs they offer, since direct competition exists from other institutions of higher learning for student clientele.

\section{Purpose, Objectives, and Hypotheses}

The purpose of this study was to identify key factors that influence the recruitment of agriculture students at land-grant and non-land-grant universities. To achieve this purpose, three research objectives, and one null hypothesis guided this investigation:

1. Describe the personal characteristics (i.e., age, academic major, a community of residence, decisions about college and major, ethnicity, gender, job considerations, previous experiences in agriculture, and school activities) influencing students' decision to pursue an agriculturally related degree;

2. Describe the external influences (i.e., significant persons, fixed college characteristics, and college efforts to communicate with students) influencing students' decision to pursue an agricultural related degree;

3. Examine differences among students' personal characteristics and external influences on their decision to pursue an agricultural related degree;

- $\mathrm{H}_{0}$ : No differences exist among students' personal characteristics and external influences on their decision to pursue an agricultural related degree.

\section{Literature Review}

Despite challenges facing the global society in terms of food and fiber, in order to accommodate an increasing population, colleges of agriculture struggle to keep pace with the demand of supplying individuals with the skills needed to navigate the industry's current and future challenges (Rocca, 2013). For example, Goecker, Smith, and Smith (2010) reported that although the number of agricultural graduates has increased slightly in recent years, more qualified individuals are urgently needed because more than $39 \%$ of positions are filled by non-agriculture graduates every year (Goecker, Smith, Fernandez, Ali, \& Goetz, 2015). Moreover, Goecker et al. (2010) reported that a $10.8 \%$ increase in jobs is expected in the U.S. labor force by 2022 , with new career opportunities in the agricultural sector accounting for more than $5 \%$ of this increase. Therefore, it is imperative that U.S. colleges of agriculture, at land-grant and non-land-grant universities, attract high caliber students to their programs of study (Rayfield, Murphey, Skaggs, \& Shaffer, 2013; 
Shrestha, Suvedi, \& Foster, 2011). Currently, colleges of agriculture offer unique pathways to equip individuals with the knowledge, skills, and dispositions needed by professionals to address the complex issues facing the global agricultural industry in the 21 st Century. Nevertheless, the problem of attracting quality individuals to the agricultural workforce appears to be intensifying.

As an illustration, the general population in the U.S. is becoming further removed from agriculture, and as a result, colleges of agriculture must place more emphasis on developing strategic communication strategies to recruit new agricultural leaders (Baker, Settle, Chiarelli, \& Irani, 2013). For example, colleges of agriculture must better understand the communication methods and techniques that resonate with today's students (Baker, Irani, \& Adams, 2011). In particular, they must examine how to facilitate sustained communicative relationships with stakeholders who have been shown to influence recruitment efforts such as donors, alumni, and parents (Smith, 2002). To accomplish this, recruiters must traverse multiple factors that have been demonstrated to influence students' academic major decisions (Chapman, 1981).

Chapman (1981) explained that factors influencing academic major decisions included: (a) influences from individuals that students perceive as important, (b) unique characteristics of the institution, and (c) communication and recruitment efforts from universities. Hossler, Braxton, and Coopersmith (1989) expanded on these findings by arguing that the confluence of the aforementioned factors not only shaped what institution students may decide to attend but also their choice of major and future job and career opportunities. However, there are other factors that affect college of agriculture's recruitment strategies (Bettis, Tackie, \& McElhenny, 2017; Calvin \& Pense, 2013). For instance, Calvin and Pense (2013) used a qualitative approach to explore barriers to recruitment for agricultural education teacher preparation programs. As a result, five findings emerged, which represented the deterrents that students experienced when deciding to pursue the major: (1) time, (2) financial issues, (3) family resistance, (4) technology, and (5) negative perceptions. As such, findings suggested that teacher preparation programs for agricultural education, and colleges of agriculture in general, must overcome numerous historical, social, and economic issues to alleviate students' concerns and ultimately encourage them to enroll in the major (Bettis et al., 2017; Lingenfelter \& Beierlein, 2006).

Given the complexities surrounding recruitment for colleges of agriculture, it is also imperative to understand how students are receiving, interpreting, and actualizing communication and recruitment efforts (Baker et al., 2013). For example, Hossler and Gallagher (1987) explained that students "may mistakenly eliminate an institution which is potentially a good choice due to a lack of awareness of the range of institutions as well as the accurate information about institutions" (p. 215). Therefore, it is imperative to promote personal, social, and career opportunities so that recruits can better understand the depth and breadth of individual departments and programs (Boumtje \& Haase-Wittler, 2007). For instance, existing evidence has demonstrated that information about individual degree programs, provided either online or through a physical copy, is the most influential source of knowledge influencing university agriculture students' academic major decisions (Bobbitt, 2006; Rocca \& Washburn, 2005). However, other studies have reported the 
importance of key personal and professional characteristics that influence students' enrollment behaviors. Such variables include: (a) career aspirations, (b) extracurricular involvement, (c) family's connection to agriculture, (d) first-generation college student status, (e) previous agricultural experience, (f) race, (g) social pressure, (h) socioeconomic status, and more (Bettis et al., 2017; Cohen, Tran, \& Suarez, 2014; Irlbeck, Adams, Akers, Burris, \& Jones, 2014; Koon, Frick, \& Igo, 2009; Rayfield, Murphrey, Skaggs, \& Shaffer, 2013; Rocca \& Washburn, 2005; Shrestha, Suvedi, \& Foster, 2011; Stair, Danjean, Blackburn, \& Bunch, 2016; Washburn, 2002; Wildman \& Torres, 2001; Williams, 2007). Overall, universities must be cognizant of the complexities that encompass student recruitment into the agricultural sciences and plan accordingly.

Further, recent generational shifts have resulted in the majority of students who are targeted for recruitment being from Generation Z, i.e., individuals born after 2000 (Howell, 2018). Generation $Z$ has different values, motivations, and views of the world than previous generational groups (Beall, 2017). As a result, differences exist regarding their academic major choices (Howell, 2018). Moreover, as globalization has intensified in recent years, Generation $\mathrm{Z}$ exhibits more diversity regarding race and ethnic identity than ever before (Beall, 2017). Therefore, understanding the factors that attract potential undergraduate students to pursue an academic major in agriculture, particularly among various demographic groups, would greatly assist colleges of agriculture in developing effective student recruitment strategies (Bettis et al., 2017; Cohen et al., 2014).

\section{Conceptual Framework}

The recruitment of undergraduate students has long piqued the interest of scholars and administrators of higher educational institutions (Bozick, 2007; Braxton, 2000; Nora, Barlow, \& Crisp, 2005; Seidman, 2005; Tinto, 1993). As a result, the literature on student recruitment is vast. Nevertheless, one of the most prominent conceptual lenses used to describe this phenomenon was developed by Chapman (1981) who distinguished key influences in his model of student success (MSS). The first factor, student characteristics, is largely dependent on individual variables such as aptitude, educational aspirations, socioeconomic status, and high school performance (Braxton \& Hirschy, 2005). However, the second factor, external influences represents the disparity among students' interest, motivation, and ability as they navigate their institution's expectations for degree completion (Chapman, 1981). Therefore, external variables include not only students' perceptions of social pressure from peers, teachers, and other relevant actors but also their views on their institution's ability to communicate its positive attributes and be financially affordable. Before students enter universities, however, the aforementioned variables are also influenced by factors such as students' (a) expectations of college life, (b) choice of college, and (c) the college's choice of the student. Given this, Chapman (1981) suggested that departure transpires after a student attends a college and perceives a lack of fit exists between their characteristics and external influences at their institution.

It should be noted that Chapman (1981) recognized existing weaknesses within the conceptual lens, especially concerning students who work or live off campus. In response, Braxton and Hirschy (2005) created a model that more accurately reflected the experiences 


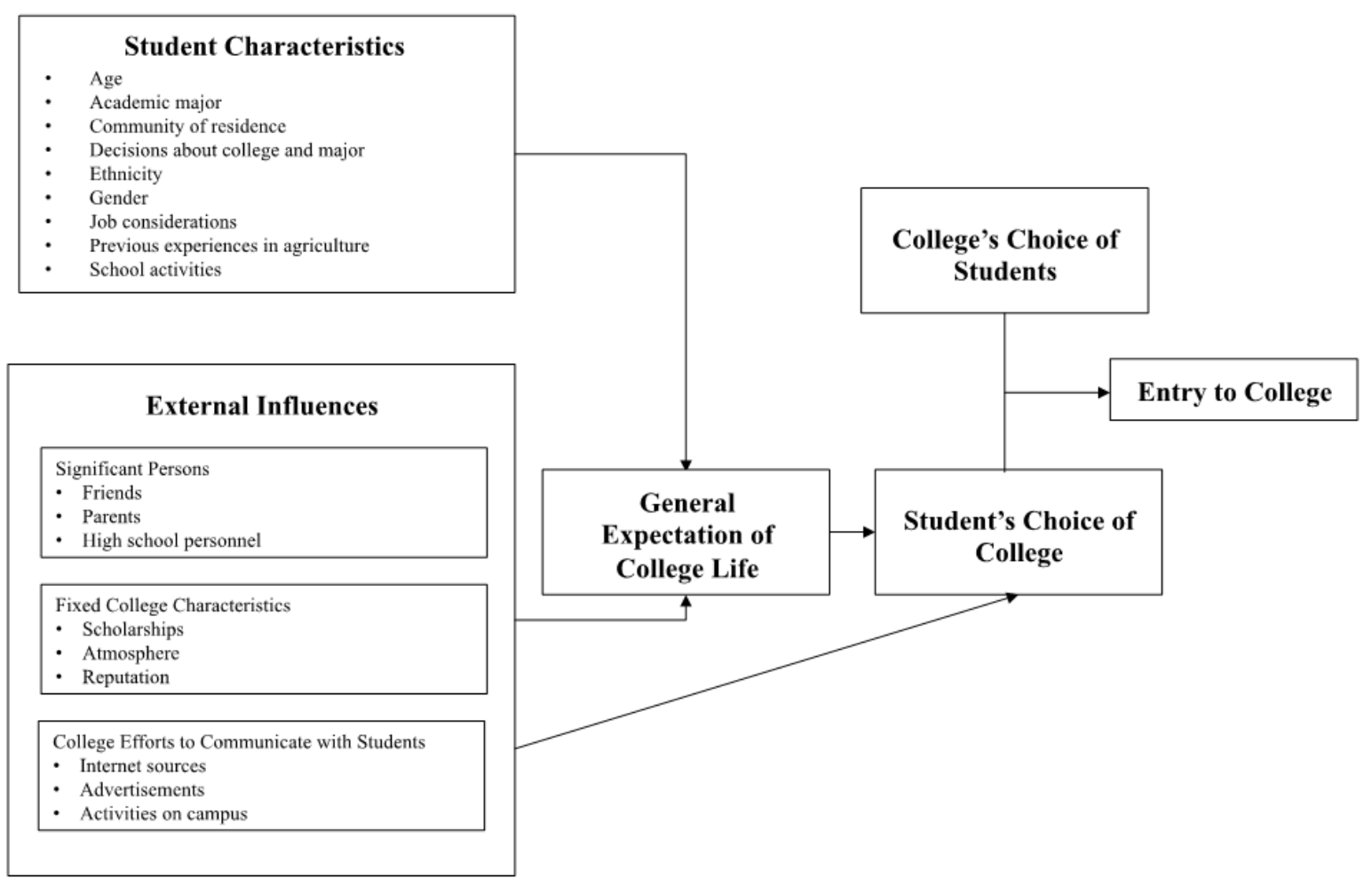

Figure 1. The conceptual framework guiding the study as modified from Chapman's (1981) MSS.

of nontraditional students in which they examined student choice using Chapman's (1981) MSS as well as various contextual factors such as: (a) age, (b) academic major, (c) community of residence, (d) decisions about college and major, (e) ethnicity, (f) gender, (g) previous experience in agriculture, and (h) school activities. Moreover, existing literature on agriculture students' academic major decisions suggests that key external variables such as significant persons, fixed college characteristics, and college's efforts to communicate with students also affects their entry to college. When viewing student success in this way, Braxton and Hirschy (2005) suggested that Chapman's (1981) model had more explanatory power and applicability. As such, we modified Chapman's (1981) MSS to include relevant contextual influences that have been reported to shape agricultural students' academic decisions. Figure 1 provides the conceptual framework guiding this investigation.

\section{Method and Procedures}

Prior to this study, no national studies had been conducted on land-grant and nonland-grant colleges of agriculture collectively, to gauge the specific strategies utilized for student recruitment and retention among existing agricultural majors to determine what 
factors encouraged them to pursue an agricultural-related major. It is important to note that data reported in this study were part of a larger investigation that examined student retention from a student, faculty, and administrative perspective. However, only data regarding students' perspective have been reported in this manuscript.

For the first part of this project, a web-based instrument was emailed to all college of agriculture academic administrators throughout the United States as listed by the Association of Public and Land-grant Universities (APLU) in accord with Dillman's, Smyth's, and Christian's (2014) tailored survey design. On the web-based instrument, administrators were asked to identify the specific strategies they utilized to recruit and retain students based on practices cited in the latest student recruitment and retention literature. Moreover, administrators indicated the extent to which those strategies were used with minority/underrepresented students. As such, a three-round web-based questionnaire approach was utilized for this study, which resulted in 58 completed surveys out of 124 (47\% return rate). For the second part of this project from which data for the current study were derived, a web-based instrument was used to analyze the factors that influenced current agricultural majors to pursue an agricultural-related major. A pre-notice message and timed reminders (Dillman et al., 2014) were sent to the 58 responding college administrators from the first part of the project, asking for them to forward the message to their respective students. This resulted in 1,015 completed surveys, representing 54 of the 58 APLU colleges. Next, we outline the specific population and measures used to analyze the factors influencing agricultural students' enrollment behaviors.

Description of the Population and Instrumentation. This study's population was a census of agriculture majors who were enrolled at universities in the APLU. The respondent frame was supplied by APLU administrators who participated in the first phase of the study. Because of this, the possibility of coverage error (Dillman et al., 2014) existed since administrators may not have forwarded the study's recruitment messages to all students who met the study's population parameters. Nevertheless, to collect data from the population, we used a web-based instrument that was slightly adapted from Rayfield's, Murphrey's, Skaggs', and Shafer's (2013) questionnaire that investigated the factors influencing college students' enrollment decisions. Rayfield et al. (2013) instrument expanded on the work of Wildman (1997) and Williams (2007). For this study, the webbased instrument was divided into three distinct sections: (1) external factors affecting students' academic major decisions, (2) student characteristics, and (3) demographic information. Items for the first section were placed on a 10-point, Likert-type scale ranging from 1 = Factor was Not Influential to $10=$ Factor was Very Influential. For the second section, items were placed on a 5-point, Like type scale using the following interpretative anchors: 1 = Never; 2 = Rarely; 3 = Sometimes; $4=$ Often, and $5=$ Always. Regarding reliability, Wildman (1997) reported a priori test-retest reliability of $75 \%$ to $100 \%$ for the instrument's first two sections. Further, the instrument's first two sections had post-hoc Cronbach's alphas at .70 or above, which is considered acceptable (Field, 2013). Before administration of the web-based instrument, it was also reviewed by a panel of experts at [State] University to establish content validity - a process resulting in only slight changes to the instrument's items to better fit the context. 
Table 1. Students' Exposure to Agriculture and Life Sciences Before College

\begin{tabular}{lllc}
\hline Items & $M$ & $S D$ & Rank \\
\hline Agriculture and life science related hobbies. & 6.87 & 3.45 & 1 \\
Personal work in an agricultural \& life sciences field of work. & 6.71 & 3.71 & 2 \\
& & & 3 \\
Agricultural science courses in high school. & 5.92 & 3.67 & 3 \\
Agricultural and life science related clubs or organizations. & 5.66 & 3.89 & 4 \\
Relatives in an agricultural and life sciences field of work. & 5.41 & 3.92 & 5 \\
4-H or FFA leadership development events. & 5.03 & 4.08 & 6 \\
4-H or FFA judging or career development events. & 4.87 & 4.06 & 7 \\
4-H or FFA livestock shows, horse shows, or rodeos. & 4.81 & 4.02 & 8 \\
Internet sources about agriculture and life sciences. & 4.36 & 2.78 & 9 \\
TV programs about agricultural and life sciences. & 4.17 & 2.73 & 10 \\
Newspaper articles about agricultural and life sciences. & 3.68 & 2.52 & 11 \\
Technical journals focused on agricultural and life sciences (e.g., & 3.55 & 2.71 & 12 \\
$\quad$ Journal of Wildlife Management, Journal of Animal Science, & & & \\
$\quad$ etc.). & & & 13 \\
Non-technical magazines about agriculture and life sciences (e.g., & 3.41 & 2.58 & \\
$\quad$ Time, US Newsweek, etc.). & & & \\
Radio broadcasts about agricultural and life sciences. & 2.96 & 2.35 & 14 \\
\hline Note. Items were placed on a 10-point, Likert-type scale ranging from 1= Not Influential to 10 & Very \\
Influential. & &
\end{tabular}

Data Analysis. The researchers used a range of descriptive and statistical analysis procedures to address the research objectives. For example, for objective one and two, descriptive statistics were analyzed to report the following measures of central tendency: (a) frequencies, (b) percentages, (c) means, and (d) standard deviations. Then, for research objectives three and four we used independent sample t-tests to analyze differences regarding students' characteristics and external influences on factors that affect their decision to pursue an agricultural related degree. Finally, effect sizes for tests of mean differences were reported using Cohen's $d$ (Field, 2013).

\section{Results}

Objective One. The first objective sought to understand students' characteristics. In total, the average participant reported they were 20.81 years old with $68.6 \%(f=696)$ identifying as female and $31.4 \%(f=319)$ reporting they were male. Only marginal differences in diversity were reported regarding participants' ethnicity; for example, 777 (76.6\%) reported they were white, 109 (10.7\%) black, 42 (4.1\%) Hispanic, 39 (3.8\%) another race, and $35(3.4 \%)$ identified as Asian/Pacific Islander. Nevertheless, more disparity existed regarding participants' community of residence with $32.8 \%(f=333)$ residing in a metropolitan area, $32.7 \%(f=332)$ a rural farm, $21.6 \%(f=220)$ in a small city or town, and $12.8 \%(f=130)$ in a rural non-farm location.

Of the students, $71.3 \%(f=724)$ entered college having already earned college credit. And participants represented a range of academic majors. For instance, the most 
Table 2. College or Departmental Factors Influencing Students' Academic Major Decisions

\begin{tabular}{lccc}
\hline Items & $M$ & $S D$ & Rank \\
\hline Friendly atmosphere in the College of Agriculture Sciences. & 6.30 & 3.36 & 1 \\
Faculty's friendliness in your department. & 6.21 & 3.34 & 2 \\
Teaching reputation of your departmental and major professors. & 6.12 & 3.50 & 3 \\
Teaching reputation of agricultural professors. & 5.87 & 3.47 & 4 \\
Internet sources about your major. & 5.50 & 3.23 & 5 \\
Agricultural related clubs/activities. & 5.12 & 3.47 & 6 \\
Personal visit with a representative from the college of agriculture & 4.63 & 3.55 & 7 \\
$\quad$ and life sciences. & 4.43 & 3.26 & 9 \\
Activities on the university campus. & 4.16 & 3.03 & 10 \\
Informational pamphlets about your major. & 4.13 & 3.42 & 11 \\
Scholarship(s) from your department & 3.87 & 3.11 & 12 \\
Advertisements about the college of agriculture. & 3.69 & 3.34 & 13 \\
Alumni from the college of agriculture and life sciences & 2.96 & 2.99 & 14 \\
High school visits from college of agriculture representatives. & 2.88 & 3.07 & 15 \\
Other financial incentives. & & \\
\hline
\end{tabular}

Note. Items were placed on a 10-point, Likert-type scale ranging from $1=$ Not Influential to $10=$ Very Influential.

frequently identified academic majors were animal science $(f=297,29.2 \%)$, agribusiness $(f=158,15.5 \%)$, agricultural education $(f=95,9.3 \%)$, environmental studies $(f=53$, $5.2 \%)$, and agricultural science $(f=43,4.2 \%)$. The majority of students $(f=550,54.2 \%)$ also reported they did not anticipate changing their major. Further, 89.7\% $(f=911)$ perceived their were ample opportunities to gain employment in their academic major area after completing their degree. Of note, however, $57 \%(f=580)$ of participants reported they did not decide to attend college until 12th grade or after they had completed high school. Similarly, 603 students $(59.4 \%)$ revealed they did not choose a college major until 12th grade or after. Participants also indicated they were engaged in an assortment of high school activities such as athletics $(f=611,60.2 \%)$, National Honor Society $(f=510$, $50.2 \%)$, school electives including debate, drama, band, and chorus $(f=451,44.4 \%)$, FFA $(f=408,40.1 \%)$, and student council or government $(f=302,29.7 \%)$.

It should also be noted the vast majority $(f=594,58.5 \%)$ of students reported they were not enrolled in agricultural education as a high school student. However, $73.6 \%$ ( $f=$ 747) of students revealed they had agricultural work experience before college in areas such as a farming and ranching $(f=456,44.9 \%)$, veterinary science $(f=187,18.4 \%)$, as well as horticulture $(f=175,17.2 \%)$. Despite largely not having previous agricultural education experience, participants explained they were predominantly aware of agricultural majors available at their university $(f=578,56.9 \%)$ as well as career opportunities associated with their current major $(f=591,58.2 \%)$ as high school students.

Nearly half $(f=502,49.5 \%)$ of participants also revealed they did not have family members with a background or experience in the agricultural industry. However, of the participants $(f=513,50.5 \%)$ who did have family members involved in agriculture, the 
Table 3. Individuals of Influence in Students' Lives Regarding Selecting an Agricultural Related Major

\begin{tabular}{lllc}
\hline Items & $M$ & $S D$ & Rank \\
\hline Parent or guardian. & 6.44 & 3.17 & 1 \\
$\quad \begin{array}{l}\text { Agricultural professional (e.g., veterinarian, rancher, wildlife } \\
\quad \text { manager, etc.). }\end{array}$ & 5.77 & 3.66 & 2 \\
$\quad$ & & & \\
$\quad \begin{array}{l}\text { Personal role model. } \\
\text { High school science teacher (e.g. biology, chemistry, earth }\end{array}$ & 4.08 & 3.94 & 3 \\
$\quad$ science, etc.). & 4.73 & 3.44 & 4 \\
Other relatives. & 4.70 & 3.33 & 5 \\
High school agriculture science teacher. & 4.19 & 3.34 & 6 \\
Friend in college. & 3.91 & 3.06 & 8 \\
Friend in high school. & 3.47 & 3.07 & 9 \\
Sister or brother. & 3.30 & 3.31 & 10 \\
Extension professional (e.g., 4-H agent or 4-H leader). & 2.77 & 2.85 & 11 \\
Other high school teacher(s) (e.g., history, math, English, etc.). & 2.53 & 2.52 & 12 \\
High school counselor. & 2.18 & 2.32 & 13 \\
High school principal or administrator. & & \\
\hline
\end{tabular}

Note. Items were placed on a 10-point, Likert-type scale ranging from $1=$ Not Influential to $10=$ Very Influential.

students most frequently reported their family had experiences in livestock production $(f=$ $260,25.6 \%)$, crop production $(f=240,23.6 \%)$, as well as previous FFA $(f=218,21.5 \%)$ and $4-\mathrm{H}$ experience $(f=203,20.0 \%)$. The students also disclosed they had a plethora of ways in which they were exposed to agricultural and life sciences before college, especially hobbies in agricultural and life sciences $(M=6.87, S D=3.45)$, personal agricultural work experiences $(M=6.71, S D=3.71)$, and agriscience education courses in high school $(M=$ $5.92, S D=3.67)$. Table 1 provides an overview of the influential sources of exposure to agriculture students received before college.

Objective Two. The second objective sought to describe the external influences that may have shaped students' decision to pursue an agricultural related degree. Of the 54 participating APLU universities, nearly half of students represented an 1862 land-grant university $(f=503,49.5 \%)$; however, $32.7 \%(f=332)$ were from a non-land-grant university, and $17.7 \%(f=180)$ attended an 1890 land-grant university. The 54 represented APLU institutions were primarily located in the southeast $(f=17,31.4 \%)$, midwest $(f=13$, $24.0 \%)$, northeast $(f=11,20.3 \%)$, and southwest $(f=11,20.3 \%)$.

Using a 10-point, Likert-type scale, students also divulged the specific college or departmental factors that most profoundly influenced their decisions regarding the selection of an academic major. Students identified the college of agriculture's friendly atmosphere $(M=6.30, S D=3.36)$, the friendliness of faculty in their academic department $(M=6.21, S D=3.34)$, and the teaching reputation of their department's professors $(M=$ $6.12, S D=3.50)$ as well as the college of agriculture's professors $(M=5.87, S D=3.47)$ most profoundly influenced their college major decisions. Table 2 provides more insight into the various college and departmental factors that influence students' decision making. 
Journal of Research in Technical Careers

Table 4. Gender's Impact on Students' Academic Major Selection Factors

\begin{tabular}{|c|c|c|c|c|c|c|}
\hline Items & Gender & $M$ & $S E$ & $t$ & $p$ & $d$ \\
\hline \multirow[t]{2}{*}{ Internet sources about agriculture \& life sciences. } & Male & 4.19 & .157 & -1.32 & .186 & .041 \\
\hline & Female & 4.44 & . 106 & & & \\
\hline \multirow[t]{2}{*}{ TV programs about agriculture \& life sciences. } & Male & 3.89 & .150 & -2.14 & $.032 *$ & .067 \\
\hline & Female & 4.29 & .104 & & & \\
\hline \multirow[t]{2}{*}{ Newspaper articles about agriculture \& life sciences. } & Male & 3.58 & .143 & -.886 & .376 & .029 \\
\hline & Female & 3.73 & .095 & & & \\
\hline \multirow[t]{2}{*}{ Radio broadcasts about agriculture \& life sciences. } & Male & 3.08 & .141 & 1.04 & .294 & .032 \\
\hline & Female & 2.91 & .086 & & & \\
\hline \multirow[t]{2}{*}{ Agricultural science courses in high school. } & Male & 5.76 & .202 & -.894 & .372 & .028 \\
\hline & Female & 5.99 & .141 & & & \\
\hline \multirow{2}{*}{$\begin{array}{l}\text { Non-technical magazines about agriculture \& life } \\
\text { sciences (e.g., Time, US News, Newsweek, etc.). }\end{array}$} & Male & 3.24 & .142 & -1.39 & .163 & .043 \\
\hline & Female & 3.48 & .099 & & & \\
\hline \multirow{2}{*}{$\begin{array}{l}\text { Technical journals focused on agriculture \& life } \\
\text { sciences (e.g., Journal of Wildlife Management, } \\
\text { Journal of Animal Science, etc.). }\end{array}$} & Male & 3.70 & .155 & 1.12 & .262 & .035 \\
\hline & Female & 3.49 & .102 & & & \\
\hline \multirow{2}{*}{$\begin{array}{l}\text { Relatives in an agricultural and life sciences field of } \\
\text { work. }\end{array}$} & Male & 5.93 & .218 & 2.86 & $.004 *$ & .089 \\
\hline & Female & 5.18 & .148 & & & \\
\hline \multirow{2}{*}{$\begin{array}{l}\text { Personal work in an agricultural \& life sciences field of } \\
\text { work. }\end{array}$} & Male & 6.78 & .207 & .454 & .650 & .014 \\
\hline & Female & 6.67 & .141 & & & \\
\hline \multirow{2}{*}{$\begin{array}{l}\text { Agriculture \& life sciences related clubs or } \\
\quad \text { organizations. }\end{array}$} & Male & 5.10 & .213 & -3.13 & $.002 *$ & .097 \\
\hline & Female & 5.92 & .148 & & & \\
\hline \multirow[t]{2}{*}{ Agriculture \& life sciences related hobbies. } & Male & 6.29 & .199 & -3.62 & $.000^{*}$ & .111 \\
\hline & Female & 7.13 & .128 & & & \\
\hline \multirow[t]{2}{*}{ 4-H or FFA leadership development events. } & Male & 4.43 & .218 & -3.20 & $.001 *$ & .100 \\
\hline & Female & 5.31 & .157 & & & \\
\hline \multirow[t]{2}{*}{ 4-H or FFA livestock shows, horse shows, or rodeos. } & Male & 3.99 & .209 & -4.40 & $.000^{*}$ & .136 \\
\hline & Female & 5.18 & .156 & & & \\
\hline \multirow[t]{2}{*}{ 4-H or FFA judging or career development events. } & Male & 4.30 & .218 & -3.02 & $.003 *$ & .094 \\
\hline & Female & 5.13 & .156 & & & \\
\hline
\end{tabular}

Note. Items were placed on a 10-point, Likert-type scale ranging from $1=$ Not Influential to $10=$ Very Influential.

Regarding the individuals that influence students' college major decisions, on average parents or guardians $(M=6.44, S D=3.17)$, agricultural professionals $(M=5.77$, $S D=3.66)$, and students' role models $(M=5.08, S D=3.94)$ were the most commonly identified. Table 3 offers insight into the individuals of influence that shape students' academic major decisions.

Objective Three. The investigation's third objective examined differences among students' personal characteristics and external influences on their decision to pursue an 
Table 5. Gender's Impact on Students' Academic Major Selection Factors (Individual People)

\begin{tabular}{|c|c|c|c|c|c|c|}
\hline Individual people & Gender & $M$ & $S E$ & $t$ & $p$ & $d$ \\
\hline \multirow[t]{2}{*}{ Parent or guardian. } & Male & 6.44 & .179 & -.037 & .970 & .001 \\
\hline & Female & 6.45 & .120 & & & \\
\hline \multirow[t]{2}{*}{ Sister or brother. } & Male & 3.31 & .162 & -1.11 & .266 & .034 \\
\hline & Female & 3.54 & .120 & & & \\
\hline \multirow[t]{2}{*}{ Other relatives. } & Male & 4.95 & .186 & 1.42 & .155 & .044 \\
\hline & Female & 4.63 & .126 & & & \\
\hline \multirow[t]{2}{*}{ Friend in high school. } & Male & 3.89 & .171 & -.169 & .866 & .027 \\
\hline & Female & 3.93 & .116 & & & \\
\hline \multirow[t]{2}{*}{ Friend in college. } & Male & 4.20 & .185 & .075 & .944 & .036 \\
\hline & Female & 4.19 & .128 & & & \\
\hline \multirow[t]{2}{*}{ Personal role model. } & Male & 5.09 & .221 & .077 & .939 & .034 \\
\hline & Female & 5.07 & .150 & & & \\
\hline \multirow{2}{*}{$\begin{array}{l}\text { High school science teacher (e.g., biology, } \\
\text { chemistry, earth science). }\end{array}$} & Male & 4.49 & .190 & -2.11 & $.034^{*}$ & .066 \\
\hline & Female & 4.98 & .131 & & & \\
\hline \multirow{2}{*}{$\begin{array}{l}\text { Extension professional (4-H agent or } 4-H \\
\text { leader). }\end{array}$} & Male & 2.93 & 169 & -2.38 & $.017 *$ & .074 \\
\hline & Female & 3.47 & .130 & & & \\
\hline \multirow[t]{2}{*}{ High school counselor. } & Male & 2.30 & .131 & -1.99 & $.046^{*}$ & .062 \\
\hline & Female & 2.64 & .099 & & & \\
\hline \multirow[t]{2}{*}{ High school agriculture science teacher. } & Male & 4.64 & .217 & -.319 & .750 & .010 \\
\hline & Female & 4.72 & .150 & & & \\
\hline \multirow{2}{*}{$\begin{array}{l}\text { Agricultural professional (e.g., veterinarian, } \\
\text { rancher, wildlife manager, etc.). }\end{array}$} & Male & 5.34 & .207 & -2.51 & $.012 *$ & .078 \\
\hline & Female & 5.96 & .138 & & & \\
\hline \multirow[t]{2}{*}{ High school principal or administrator. } & Male & 2.37 & 2.37 & 1.74 & .082 & .054 \\
\hline & Female & 2.09 & 2.09 & & & \\
\hline \multirow{2}{*}{$\begin{array}{l}\text { Other high school teacher(s) (e.g., history, } \\
\text { math, English, etc.). }\end{array}$} & Male & 2.67 & 2.67 & -.735 & .462 & .023 \\
\hline & Female & 2.82 & 2.82 & & & \\
\hline
\end{tabular}

Note. Items were placed on a 10-point, Likert-type scale ranging from $1=$ Not Influential to $10=$ Very Influential.

agricultural related degree. To accomplish this, we used appropriate tests of mean comparisons, i.e., ANOVA and t-test, on the variables of interest. In particular, through the use of t-test analysis procedures, it was determined that females had a statistically significant difference $(p<.05)$ from males regarding student's academic major selection factors regarding TV programs about agriculture and life science $(t=-2.14, p=.032, \mathrm{~d}=$ $.041)$, agriculture and life science related clubs and organizations $(t=-3.13, p=.002, d=$ $.097)$, agricultural and life sciences related hobbies $(t=-3.62, p=.000, d=.111), 4-\mathrm{H}$ or FFA leadership development events $(t=-3.20, p=.001, d=1.00), 4-\mathrm{H}$ or FFA livestock shows, horse shows, or rodeos $(t=-3.20, p=.001, d=1.00)$, and 4-H or FFA judging or career development events $(t=-3.02, p=.003, d=.094)$. Further, there is statistically significant differences $(p<.05)$ between females and males were also found regarding the 
Table 6. Gender Impact upon College Based Major Selection Factors (College-Based Factors)

\begin{tabular}{|c|c|c|c|c|c|c|}
\hline Items & Gender & $M$ & $S E$ & $t$ & $p$ & $d$ \\
\hline \multirow{2}{*}{$\begin{array}{l}\text { Alumni from the college of agriculture and life } \\
\text { sciences. }\end{array}$} & Male & 3.88 & .194 & 1.20 & .230 & .037 \\
\hline & Female & 3.61 & .124 & & & \\
\hline \multirow[t]{2}{*}{ Scholarship(s) from your department. } & Male & 4.05 & .185 & -.535 & .593 & .016 \\
\hline & Female & 4.17 & .132 & & & \\
\hline \multirow[t]{2}{*}{ Other financial incentives. } & Male & 3.00 & .174 & .842 & .400 & .026 \\
\hline & Female & 2.82 & .116 & & & \\
\hline \multirow[t]{2}{*}{ Informational pamphlets about your major. } & Male & 3.75 & .162 & -2.94 & $.003^{*}$ & .092 \\
\hline & Female & 4.35 & .117 & & & \\
\hline \multirow{2}{*}{$\begin{array}{l}\text { Personal visit with a representative from college } \\
\text { of agriculture. }\end{array}$} & Male & 4.19 & .186 & -2.63 & $.009 *$ & .082 \\
\hline & Female & 4.83 & .138 & & & \\
\hline \multirow[t]{2}{*}{ Faculty's friendliness in your department. } & Male & 6.00 & .192 & -1.29 & .196 & .040 \\
\hline & Female & 6.30 & .130 & & & \\
\hline \multirow{2}{*}{$\begin{array}{l}\text { High school visits from college of agriculture } \\
\text { representatives. }\end{array}$} & Male & 2.77 & .161 & -1.36 & .174 & .042 \\
\hline & Female & 3.04 & .115 & & & \\
\hline \multirow{2}{*}{$\begin{array}{l}\text { Friendly atmosphere in college of agriculture } \\
\text { sciences. }\end{array}$} & Male & 6.08 & .191 & -1.40 & .160 & .043 \\
\hline & Female & 6.40 & .127 & & & \\
\hline \multirow{2}{*}{ Teaching reputation of agricultural professors. } & Male & 5.91 & .193 & .293 & .770 & .009 \\
\hline & Female & 5.84 & .132 & & & \\
\hline \multirow{2}{*}{$\begin{array}{l}\text { Teaching reputation of your departmental \& } \\
\text { major professors. }\end{array}$} & Male & 6.08 & .196 & -.207 & .836 & .006 \\
\hline & Female & 6.13 & .133 & & & \\
\hline \multirow[t]{2}{*}{ Internet sources about your major. } & Male & 5.02 & .180 & -3.23 & $.001^{*}$ & .100 \\
\hline & Female & 5.72 & .122 & & & \\
\hline \multirow[t]{2}{*}{ Advertisements about the college of agriculture. } & Male & 3.56 & .169 & -2.12 & $.034 *$ & .066 \\
\hline & Female & 4.01 & .119 & & & \\
\hline \multirow[t]{2}{*}{ Agricultural related clubs/activities. } & Male & 4.34 & .187 & -4.93 & $.000^{*}$ & .153 \\
\hline & Female & 5.48 & .132 & & & \\
\hline \multirow[t]{2}{*}{ Activities on the university campus. } & Male & 3.88 & .175 & -3.62 & $.000^{*}$ & .113 \\
\hline & Female & 4.68 & .125 & & & \\
\hline
\end{tabular}

Note. Items were placed on a 10-point, Likert-type scale ranging from $1=$ Not Influential to $10=$ Very Influential.

impact of relatives in an agricultural and life science field of work $(t=2.86, p=.004, d$ $=.089$ ) (see Table 4).

Statistically significant $(p<.05)$ differences were also discovered between females and males regarding the impact of individuals that influenced their selection of an academic major. For example, it was revealed that, on average, females identified the following individuals as more influential than males: (a) high school science teachers $(t=-2.11, p=$ $.034, d=.066)$, (b) extension professionals $(t=-2.38, p=.017, d=.074)$, (c) high school counselors $(t=-1.99, p=.046, d=.062)$, and (d) agricultural professionals $(t=-2.51, p=$ $.012, d=.078)$. No other individuals of influence were found to have statistically significant 
Table 7. Race/Ethnicity's Impact on Sources of Agricultural Information that Influenced Students' Academic Major Selection Factors

\begin{tabular}{|c|c|c|c|c|c|c|}
\hline Items & $\begin{array}{l}\text { Race/ } \\
\text { Ethnicity }\end{array}$ & $M$ & $S E$ & $t$ & $p$ & $d$ \\
\hline \multirow[t]{2}{*}{ Internet sources about agriculture \& life sciences. } & Minority & 4.80 & .204 & 2.67 & $.008 *$ & .083 \\
\hline & Majority & 4.24 & .096 & & & \\
\hline \multirow[t]{2}{*}{ TV programs about agriculture \& life sciences. } & Minority & 4.57 & .193 & 2.52 & $.012 *$ & .079 \\
\hline & Majority & 4.05 & .096 & & & \\
\hline \multirow[t]{2}{*}{ Newspaper articles about agriculture $\&$ life sciences. } & Minority & 3.58 & .176 & -.689 & .491 & .021 \\
\hline & Majority & 3.71 & .089 & & & \\
\hline \multirow[t]{2}{*}{ Radio broadcasts about agriculture \& life sciences. } & Minority & 2.88 & .164 & .668 & .551 & .021 \\
\hline & Majority & 2.98 & .083 & & & \\
\hline \multirow[t]{2}{*}{ Agricultural science courses in high school. } & Minority & 5.38 & .242 & .774 & $.013 *$ & .024 \\
\hline & Majority & 6.07 & .131 & & & \\
\hline \multirow{2}{*}{$\begin{array}{l}\text { Non-technical magazines about agriculture \& life sciences } \\
\quad \text { (Time, US News, Newsweek, etc.). }\end{array}$} & Minority & 3.66 & .181 & .239 & .084 & .007 \\
\hline & Majority & 3.32 & .091 & & & \\
\hline \multirow{2}{*}{$\begin{array}{l}\text { Technical journals focused on agriculture \& life sciences } \\
\text { (Journal of Wildlife Management, Journal of Animal } \\
\text { Science, etc.). }\end{array}$} & Minority & 3.76 & .196 & 1.23 & .217 & .038 \\
\hline & Majority & 3.50 & .095 & & & \\
\hline \multirow[t]{2}{*}{ Relatives in an agricultural \& life sciences field of work. } & Minority & 4.01 & .249 & 1.16 & $.000 *$ & .036 \\
\hline & Majority & 5.82 & .139 & & & \\
\hline \multirow{2}{*}{$\begin{array}{l}\text { Personal work in an agricultural \& life sciences field of } \\
\text { work. }\end{array}$} & Minority & 5.46 & .253 & -5.79 & $.000 *$ & .179 \\
\hline & Majority & 7.07 & .129 & & & \\
\hline \multirow[t]{2}{*}{ Agriculture \& life sciences related clubs or organizations. } & Minority & 4.58 & .252 & -4.80 & $.000^{*}$ & .149 \\
\hline & Majority & 5.98 & .138 & & & \\
\hline \multirow[t]{2}{*}{ Agriculture \& life sciences related hobbies. } & Minority & 6.10 & .237 & -3.81 & $.000 *$ & .119 \\
\hline & Majority & 7.09 & .122 & & & \\
\hline \multirow[t]{2}{*}{ 4-H or FFA leadership development events. } & Minority & 3.72 & .240 & -5.51 & $.000 *$ & .171 \\
\hline & Majority & 5.40 & .147 & & & \\
\hline \multirow[t]{2}{*}{ 4-H or FFA livestock shows, horse shows, or rodeos. } & Minority & 3.64 & .241 & -5.02 & $.000 *$ & .156 \\
\hline & Majority & 5.16 & .146 & & & \\
\hline \multirow[t]{2}{*}{ 4-H or FFA judging or career development events. } & Minority & 3.52 & .240 & -5.69 & $.000 *$ & .176 \\
\hline & Majority & 5.25 & .147 & & & \\
\hline
\end{tabular}

Note. Items were placed on a 10-point, Likert-type scale ranging from $1=$ Not Influential to $10=$ Very Influential.

$(p>.05)$ differences in regard to gender. Table 5 details the impact of gender on how students view influential people when making academic major decisions.

Regarding specific college-based factors, results demonstrated that gender also had a statistically significant $(p<.05)$ impact on the value students placed on specific recruitment methods (Table 6). For example, on average females had statistically significant differences from males on their views regarding informational pamphlets about their major $(t=-2.94, p=.003, d=.092)$, personal visits with representatives from the college $(t=-2.63, p=.009, d=.082)$, internet sources about the major $(t=-3.23, p=.001$, $d=.100)$, advertisements about the college of agriculture $(t=-2.12, p=.034, d=.066)$, agricultural related clubs and activities $(t=-4.93, p=.000, d=.153)$, and activities on the university campus $(t=-3.62, p=.000, d=.113)$. 
Table 8. Ethnicity's Impact on Students' Academic Major Selection Factors (Individual People Factor)

\begin{tabular}{|c|c|c|c|c|c|c|}
\hline Items & $\begin{array}{l}\text { Race/ } \\
\text { Ethnicity }\end{array}$ & $M$ & $S D$ & $t$ & $p$ & $d$ \\
\hline \multirow[t]{2}{*}{ Parent or guardian. } & Minority & 5.76 & .226 & -3.73 & $.000 *$ & .116 \\
\hline & Majority & 6.65 & .110 & & & \\
\hline \multirow[t]{2}{*}{ Sister or brother. } & Minority & 3.43 & .212 & -.279 & .781 & .008 \\
\hline & Majority & 3.49 & .109 & & & \\
\hline \multirow[t]{2}{*}{ Other relatives. } & Minority & 4.31 & .225 & -2.24 & $.025^{*}$ & .070 \\
\hline & Majority & 4.87 & .119 & & & \\
\hline \multirow[t]{2}{*}{ Friend in high school. } & Minority & 3.76 & .208 & -.924 & .355 & .029 \\
\hline & Majority & 3.97 & .109 & & & \\
\hline \multirow[t]{2}{*}{ Friend in college. } & Minority & 4.16 & .227 & -.213 & .832 & .006 \\
\hline & Majority & 4.22 & .119 & & & \\
\hline \multirow[t]{2}{*}{ Personal role model. } & Minority & 4.56 & .253 & 2.32 & $.020^{*}$ & .072 \\
\hline & Majority & 5.25 & .142 & & & \\
\hline \multirow{2}{*}{$\begin{array}{l}\text { High school science teacher (e.g., biology, } \\
\text { chemistry, earth science.) }\end{array}$} & Minority & 4.71 & .232 & -.567 & .571 & .017 \\
\hline & Majority & 4.85 & .123 & & & \\
\hline \multirow{2}{*}{$\begin{array}{l}\text { Extension professional (e.g., 4-H agent or 4-H } \\
\quad \text { leader). }\end{array}$} & Minority & 2.83 & .207 & -2.42 & $.016^{*}$ & .076 \\
\hline & Majority & 3.44 & .120 & & & \\
\hline \multirow[t]{2}{*}{ High school counselor. } & Minority & 2.79 & .189 & 1.79 & .074 & .056 \\
\hline & Majority & 2.45 & .087 & & & \\
\hline \multirow[t]{2}{*}{ High school agriculture science teacher } & Minority & 3.74 & .243 & -4.19 & $.000 *$ & .130 \\
\hline & Majority & 4.97 & .142 & & & \\
\hline \multirow{2}{*}{$\begin{array}{l}\text { Agricultural professional (e.g., veterinarian, } \\
\text { rancher, wildlife manager, etc.). }\end{array}$} & Minority & 5.08 & .244 & -3.29 & $.001^{*}$ & .103 \\
\hline & Majority & 5.98 & .130 & & & \\
\hline \multirow[t]{2}{*}{ High school principal or administrator. } & Minority & 2.05 & .143 & -.911 & .363 & .028 \\
\hline & Majority & 2.21 & .085 & & & \\
\hline \multirow{2}{*}{$\begin{array}{l}\text { Other high school teacher(s) (e.g., history, } \\
\text { math, English) }\end{array}$} & Minority & 2.04 & .195 & .273 & .334 & .008 \\
\hline & Majority & 2.23 & 2.84 & & & \\
\hline
\end{tabular}

T-test analysis was also used to examine differences regarding the role that various sources have on students' academic major selection based on whether they self-identified as belonging to a (1) a majority race/ethnicity group, i.e., Caucasian students, and (2) a minority race/ethnic group, i.e., students identifying as black, Asian, multiple races, and more. Based on this analysis, results demonstrated that statistically significant $(p<.05)$ differences existed regarding minority students' reporting on the importance of internet sources about agricultural and life sciences $(t=2.67, p=.008, d=.083)$ and TV programs about agricultural and life sciences $(t=2.52, p=.012, d=.079)$ had in shaping their academic major decisions. However, students who identified as belonging to a majority race/ethnic group demonstrated statistically significant $(p<.05)$ differences regarding the following sources in influencing their academic major decisions: (a) agricultural science courses in high school $(t=.774, p=.013, d=.024)$, (b) relatives in an agricultural and life 
Table 9. Ethnicity/Race's Impact on Students' Academic Major Selection (College-based Factors)

\begin{tabular}{|c|c|c|c|c|c|c|}
\hline Items & $\begin{array}{l}\text { Race/ } \\
\text { Ethnicity }\end{array}$ & $M$ & $S E$ & $t$ & $p$ & $d$ \\
\hline \multirow{2}{*}{$\begin{array}{l}\text { Alumni from the college of agriculture and life } \\
\text { sciences. }\end{array}$} & Minority & 3.29 & .212 & -2.07 & $.038 *$ & .065 \\
\hline & Majority & 3.81 & .121 & & & \\
\hline \multirow[t]{2}{*}{ Scholarship(s) from your department. } & Minority & 4.21 & .233 & .464 & .643 & .014 \\
\hline & Majority & 4.09 & .121 & & & \\
\hline \multirow[t]{2}{*}{ Other financial incentives. } & Minority & 3.29 & .218 & 2.31 & $.021 *$ & .072 \\
\hline & Majority & 2.76 & .107 & & & \\
\hline \multirow[t]{2}{*}{ Informational pamphlets about your major. } & Minority & 4.59 & .211 & 2.41 & $.016^{*}$ & .075 \\
\hline & Majority & 4.04 & .107 & & & \\
\hline \multirow{2}{*}{$\begin{array}{l}\text { Personal visit with a representative from } \\
\text { college of agriculture. }\end{array}$} & Minority & 4.51 & .241 & -.543 & .588 & .017 \\
\hline & Majority & 4.65 & .126 & & & \\
\hline \multirow[t]{2}{*}{ Faculty’s friendliness in your department. } & Minority & 5.94 & .237 & -.1 .26 & .205 & .039 \\
\hline & Majority & 6.27 & .122 & & & \\
\hline \multirow{2}{*}{$\begin{array}{l}\text { High school visits from college of agriculture } \\
\text { representatives. }\end{array}$} & Minority & 2.82 & .201 & .201 & .431 & .006 \\
\hline & Majority & 3.00 & .107 & & & \\
\hline \multirow{2}{*}{$\begin{array}{l}\text { Friendly atmosphere in college of agriculture } \\
\text { sciences. }\end{array}$} & Minority & 5.79 & .239 & -2.54 & $.011 *$ & .079 \\
\hline & Majority & 6.44 & .118 & & & \\
\hline \multirow[t]{2}{*}{ Teaching reputation of agricultural professors. } & Minority & 5.50 & .243 & -1.82 & .068 & .057 \\
\hline & Majority & 5.98 & .122 & & & \\
\hline \multirow{2}{*}{$\begin{array}{l}\text { Teaching reputation of your departmental \& } \\
\text { major professors. }\end{array}$} & Minority & 5.75 & .244 & -1.86 & .063 & .058 \\
\hline & Majority & 6.24 & .123 & & & \\
\hline \multirow[t]{2}{*}{ Internet sources about your major. } & Minority & 5.70 & .223 & 1.07 & .283 & .033 \\
\hline & Majority & 5.44 & .114 & & & \\
\hline \multirow[t]{2}{*}{ Advertisements about college of agriculture. } & Minority & 3.99 & .216 & .648 & .517 & .020 \\
\hline & Majority & 3.83 & .110 & & & \\
\hline \multirow[t]{2}{*}{ Agricultural related clubs/activities. } & Minority & 4.74 & .234 & -1.91 & .056 & .060 \\
\hline & Majority & 5.24 & .124 & & & \\
\hline \multirow[t]{2}{*}{ Activities on the university campus. } & Minority & 4.36 & .218 & -.383 & .702 & .012 \\
\hline & Majority & 4.45 & .117 & & & \\
\hline
\end{tabular}

Note. Items were placed on a 10-point, Likert-type scale ranging from $1=$ Not Influential to $10=$ Very Influential .

science field of work $(t=1.16, p=.000, d=.036)$, (c) personal work experiences in agricultural and life sciences $(t=-5.79, p=.000, d=.179)$, (d) agricultural and life sciences related clubs and organizations $(t=-4.80, p=.000, d=.149)$, agricultural and life sciences related hobbies $(t=-3.81, p=.000, d=.119)$, 4-H or FFA leadership development events $(t=-5.51, p=.000, d=.171), 4$-H or FFA livestock shows, horse shows, or rodeos $(t=-$ $5.02, p=.000, d=.156)$, and 4-H or FFA judging or career development events $(t=-5.69$, $p=.000, d=.176)$. The remaining sources tested did not demonstrate statistically significant $(p>.05)$ differences (see Table 7). 
Journal of Research in Technical Careers

Differences regarding the importance of specific individuals in majority and minority students' academic major decisions were also tested. On average, minority students reported statistically significant $(p<.05)$ differences concerning the importance of their parent or guardian $(t=-3.73, p=.000, d=.116)$ in influencing their academic major decisions compared to students who identified as belonging to a majority race/ethnicity group. On the other hand, majority students demonstrated statically significant $(p<.05)$ differences regarding the influence of other relatives $(t=-2.24, p=.025, d=.070)$, personal role models $(t=2.32, p=.020, d=.072)$, extension professionals $(t=-2.42, p=.016, d=$ $.076)$, high school agricultural science teachers $(t=-4.19, p=.000, d=.103)$, and other agricultural professionals $(t=-3.29, p=.001, d=.103)$. The remaining seven individuals of influence identified through the literature did not yield statistically significant $(p>.05)$ differences (see Table 8).

Students who identified as minority and majority students also exhibited statistically significant $(p<.05)$ differences in how they viewed the importance of a range of college-based factors on their academic major decisions. For instance, in general, minority students reported they placed more value on three key sources than majority students: (1) alumni from the college and life science $(t=-2.07, p=.038, d=.065)$, (2) financial incentives $(t=2.31, p=.021, d=.072)$, and informational pamphlets about the major $(t=2.41, p=.016, d=.075)$. Meanwhile, students who identified as representing a majority group placed more value on the friendly atmosphere in the college of agricultural and life sciences $(t=-2.54=.011, d=.079)$. Table 9 provides a detailed overview of the differences between majority and minority students based on college-based factors that affected their decision regarding their academic major.

\section{Conclusions, Recommendations, and Implications}

This national study sought to describe key factors that influenced the recruitment of agriculture students at land-grant and non-land-grant universities. In the investigation, nearly $70 \%(68.6 \% ; f=696)$ of participants were female, suggesting that women could play a more prominent role in addressing complex issues and problems facing the agricultural industry in the future (United Nations, 2015). Nevertheless, it is important to understand if female students are successfully attaining and staying in agricultural related careers after earning a degree. Therefore, we recommend that future research analyze trends concerning women's successful entry into agricultural careers as well as attrition rates of females in such positions.

With only a $22.1 \%(f=225)$ racial minority representation in this study, it is apparent that more work is needed to attract individuals from a range of cultural and ethnic backgrounds who hold baccalaureate degrees in agriculture. Further, of the students, nearly $30 \%(f=297,29.2 \%)$ were animal science majors - the largest represented academic major. Therefore, the researchers, recommend that additional effort be placed on recruiting a diverse pool of students to agriculture's various sectors and corresponding academic majors that will be vital to producing enough food, fuel, and fiber to respond to global demands by 2050 (United Nations, 2015). 
It was also determined that 12th grade was pivotal in students' college decision making $(f=580 ; 57 \%)$ as well their future academic major $(f=603 ; 59.4 \%)$. Such findings support existing literature (Herren, 2005; Rocca \& Washburn, 2005) reported on agricultural recruitment. As such, we recommend that colleges of agriculture increase communication and education about agricultural related academic majors and careers in the months before and during students' senior year of high school. For example, more open house events, internet-based information, targeted social media messages, and financial and scholarship materials should be distributed to ensure students have the resources they need to make informed decisions about their future.

Through our analysis of the data, it was also determined that differences existed between students' personal characteristics and external influences on their decision to pursue an agricultural related degree. Therefore, the null hypothesis for the investigation was rejected. In particular, we noted statistically significant $(p<.05)$ differences between females and males existed concerning the importance of seven factors in shaping their academic major decisions. Those factors included: (1) TV programs about agriculture and life science, (2) agriculture and life science related clubs and organizations, (3) agricultural and life sciences related hobbies, (4) 4-H or FFA leadership development events, (5) 4-H or FFA livestock shows, horse shows, or rodeos, (6) 4-H or FFA judging or career development events, and (7) relatives in an agricultural and life science field of work. Although existing literature has noted the importance of agricultural related experiences (Bradford, 2017; Herren, 2005), differences regarding gender on such factors is scarce in existing agricultural recruitment literature. Given that it appears that gender plays a key role in how students interpret the importance of select direct and vicarious experiences in shaping their academic and career decisions about agriculture, colleges of agriculture should more deeply understand how to target students based on these factors. As such, we recommend that future investigations use a qualitative approach to distill why such differences exist while also providing implications for how colleges of agriculture can better navigate such complexities moving forward.

As a result of this investigation, we also found statistically significant $(p<.05)$ differences between females and males regarding the impact of individuals of influence on their academic major decisions, a finding that is supported by existing work (Bobbit, 2006; Williams \& Warren, 2014). For example, on average females reported the following individuals had more influence on their decisions than their male counterparts: (a) high school science teachers, (b) extension professionals, (c) high school counselors, and (d) agricultural professionals. Therefore, more work is needed to educate such individuals about degree options, career pathways, and other opportunities in colleges of agriculture. Further, additional research should explore the communication methods from colleges of agriculture that resonate best with individuals that have been identified to influence females' academic major decisions.

We also conclude that statistically significant $(p<.05)$ differences existed between male and females regarding the value they place on specific recruitment methods - a finding not supporting by existing literature. Generally, females placed more value on informational pamphlets about their major, personal visits with representatives from the college, internet sources about the major, advertisements about the college of agriculture, 
agricultural-related clubs and activities, and activities on the university campus. As such, we recommend more emphasis on such methods by tailoring them specifically to female recruits. Despite this insight into successful strategies to recruit female students, our analysis did not identify any statistically significant $(p>.05)$ recruitment methods that males placed more value on than females. Therefore, we recommend that future research explore new, novel ways to recruit male students into agricultural majors, considering that males only comprised $31.4 \%(f=319)$ of participants in this study.

We also conclude that statistically significant $(p<.05)$ differences existed between minority and majority students concerning the role that various sources of agricultural information had in influencing their decision to major in an agriculturally related degree. For example, minority students identified that internet sources and TV programs about agricultural and life sciences were critical sources during their decision-making process. Currently, a dearth of knowledge exists regarding the sources of information that influence minority students' academic degree decisions. As such, future investigations should more purposefully explore the types of web and TV-based content that affect students' academic major decisions. Further, generative discussions and outreach efforts could also result from a moment-to-moment analysis of the types of web, video, and audio recruitment content that most profoundly resonates with minority students. This study also revealed that majority students, i.e., Caucasian, placed more value on the following experiences and contacts than minority students: (a) agricultural science courses in high school, (b) relatives in an agricultural and life science field of work, (c) personal work experiences in agricultural and life sciences, (d) agricultural and life sciences related clubs and organizations, (e) agricultural and life sciences related hobbies, (f) 4-H or FFA leadership development events, (g) 4-H or FFA livestock shows, horse shows, or rodeos, and (h) 4-H or FFA judging or career development events. Therefore, additional effort should be made during students' primary and secondary schooling to emphasize experience, personal connections, and literacy in agriculture.

We also discovered statistically significant $(p<.05)$ differences between minority and majority students regarding the individuals who influence their decisions about academic majors. For example, on average minority students reported their parent or guardian influenced their decisions more than majority students. As such, when recruiting minority students, we recommend that representatives from colleges of agriculture take time to form a personal connection with parents and guardians and alleviate any uncertainty they may have about their child pursuing a degree in agriculture. Majority students, however, suggested that other relatives, personal role models, extension professionals, high school agricultural science teachers, and agricultural professionals influenced their decisions more than minority students. Given this, we recommend that recruitment campaigns and materials be expanded to include individuals of influence so they can help students understand the various opportunities available.

We also conclude that minority students demonstrated statistically significant $(p<$ .05) differences from majority students regarding the importance they placed on several college-based factors - a finding not currently reflected in the literature. For example, on average minority students identified the importance of alumni, financial incentives, and informational pamphlets about the major more than more majority students. Perhaps, 


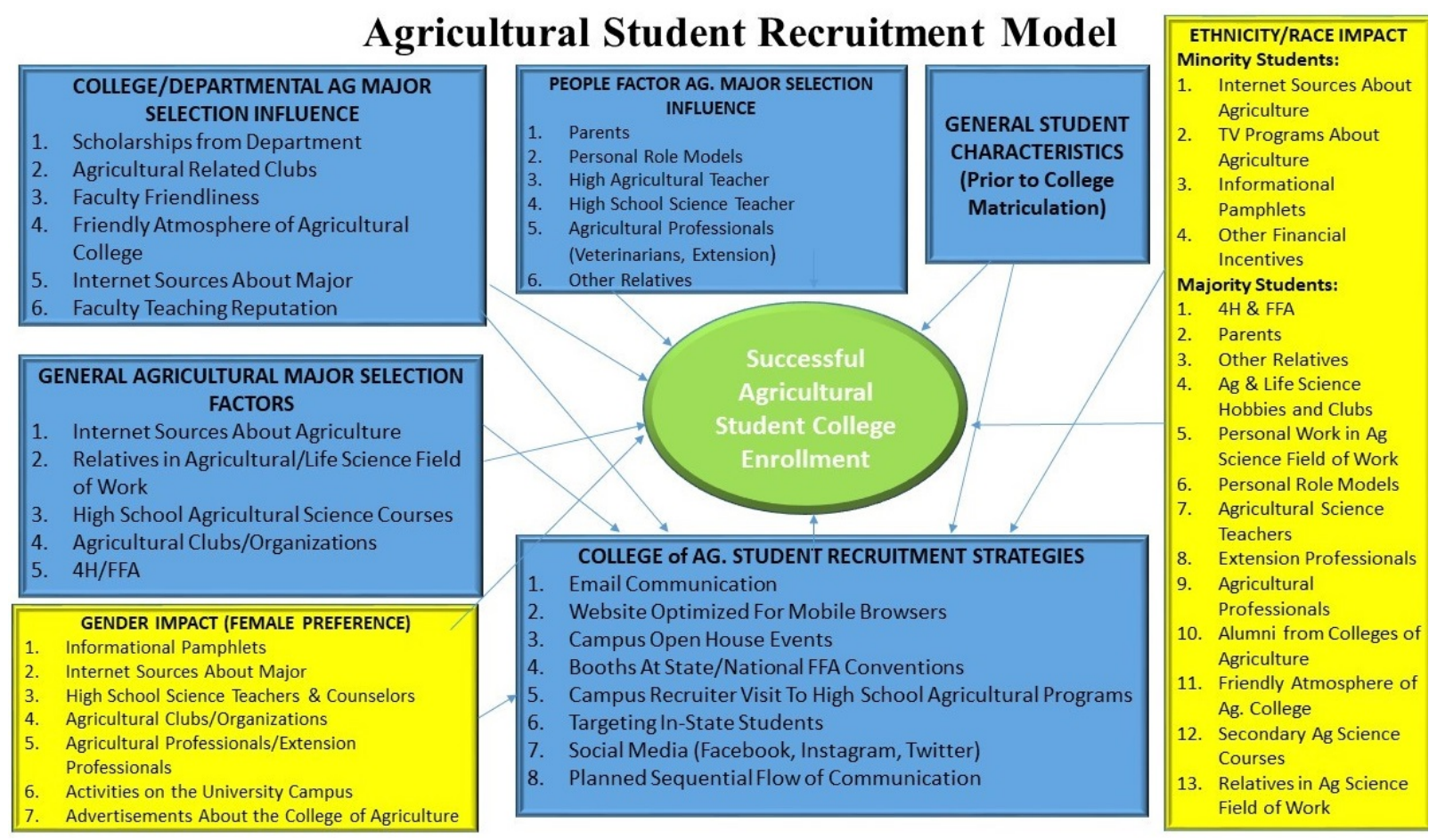

Figure 2. Agricultural student recruitment model.

stories from minority program alumni and information concerning scholarships, workstudy programs, research apprenticeships, and other relevant opportunities should be distributed to minority students through more focused recruitment strategies. Further, additional qualitative research is needed to understand better the types of stories and interactions from program alumni that motivate minority students to pursue a degree in agriculture. On the other hand, majority of students more readily acknowledged the importance of the college of agricultural and life sciences having a friendly atmosphere in influencing their academic major decisions. As such, colleges of agriculture should consider alternative ways to have faculty and staff interact with potential students so they can better determine whether they will have a positive experience after enrollment. Finally, we conclude that statistically significant $(p<.05)$ differences were present between minority students and all other students regarding their views on successful recruitment efforts facilitated by agricultural diversity offices. In particular, minority students identified email and social media posts as successful recruitment methods. Therefore, the researchers recommend that quality web-based communication and recruitment campaigns be developed to recruit more minority students into colleges of agriculture in the future.

\section{Agricultural Student Recruitment Model}


Currently, the agricultural industry struggles to fill positions with qualified agricultural workers (Goecker et al., 2015). It is critical, therefore, to attract high caliber individuals to agricultural degree programs that are prepared to address complex issues and problems. Through the current investigation, we illuminated a number of indicators that affect students' decision to pursue an agricultural degree at land-grant and non-land-grant universities across the U.S. We also provided a deeper understanding of key differences among these factors from a gendered and minority student perspective, and as a result, we now have a better understanding of how to design and deliver tailored recruitment campaigns based on a range student diversity factors. To operationalize findings for U.S. colleges of agriculture, we developed the agricultural student recruitment model (ASRM). The model visually represents the distinct but intersecting factors that most profoundly influence students' decisions. The factors identified in this study that were found to foreground successful agricultural student enrollment included: (a) college and departmental agricultural major selection influences, (b) general agricultural major selection factors, (c) gender impacts, (d) people of significance, (e) college of agriculture recruitment strategies (data taken from the companion study to this one, regarding agricultural academic administrators most effective recruitment strategies), (f) general student characteristics, and (g) ethnicity/race impacts (Figure 2). We recommend colleges of agriculture use the ASRM as a tool when they consider ways to better resonate with populations that lack representation in particular degree programs as well as their state's agricultural industry. Through this focused recruitment strategy, perhaps greater advancements can be made to supply a workforce that is more reflective of the general population and also prepared to navigate the agricultural industry's grand challenges in the future.

\section{References}

Baker, L. M., Irani, T., \& Abrams, K. (2011). Communicating strategically with generation me: Aligning students' career needs with communication about academic programs and available careers. NACTA Journal, 55(2), 32-39. Retrieved from https://www.nactateachers.org/attachments/article/1148/Baker_JUNE\%202011\%20NAC TA\%20Journal-8.pdf

Baker, L. M., Settle, Q., Chiarelli, C., \& Irani, T. (2013). Recruiting strategically: Increasing enrollment in academic programs of agriculture. Journal of Agricultural Education, 54(3), 54. https://doi.org/10.5032/jae.2013.03054

Beall, G. (2017). Eight key difference between Generation Z and Millennials. New York, NY: Huffington Post. Retrieved from https://www.huffpost.com/entry/8-key-differencesbetween_b_12814200

Bettis, J. L., Sr., Tackie, D. N. O., McElhenney, W. H., \& Bettis, J. L., Jr. (2017). Factors which influence African Americans' decisions to choose the forestry/natural resources management professions: The Tuskegee University case. NACTA Journal, 61(3), 248254. Retrieved from https://www.nactateachers.org/attachments/article/2624/15\%20\%20Bettis.pdf 
Bobbitt, R. K. (2006). Factors influencing recruitment, retention, and job placement in the College of Agricultural Sciences and Natural Resources at Texas Tech (Master's thesis). Lubbock, TX: Texas Tech University.

Boumtje, P., \& Haase-Wittler, P. S. (2007). Factors affecting enrollment of minority students in agriculture majors at Southern Arkansas University. Proceedings from 2007 CTE Research and Professional Development Conference, Las Vegas, $\mathrm{NV}$.

Bozick, R. (2007). Making it through the first year of college: The role of students' economic resources, employment, and living arrangements. Sociology of Education, 80(3), 261285. https://doi.org/10.1177/003804070708000304

Bradford, J. S. (2017). College choice: Perceptions of first-year students in the College of Agricultural Sciences and Natural Resources at Oklahoma State University (Master's thesis). Stillwater, OK: Oklahoma State University.

Braxton, J. M. (2000). Reworking the student departure puzzle. Nashville, TN: Vanderbilt University Press.

Braxton, J. M., \& Hirschy, A. S. (2005). Theoretical developments in the study of college student departure. Washington, DC; American Council on Education

Calvin, J., \& Pense, S. L. (2013). Barriers and solutions to recruitment strategies of students into post-secondary agricultural education programs: A focus group approach. Journal of Agricultural Education, 54(4), 45-57. https://doi.org/10.5032/jae.2013.04045

Chapman, D. W. (1981). A model of student college choice. The Journal of Higher Education, 52(5), 490-505. Retrieved from https://doi.org/10.1080/00221546.1981.11778120

Dillman, D. A., Smyth, J. D., \& Christian, L. M. (2014). Internet, phone, mail, and mixed-mode surveys: The tailored design method (4th ed.). Hoboken, NJ: John Wiley \& Sons.

Food and Agriculture Organization of The United Nations (2017). The future of food and agriculture Trends and challenges. Rome: The United Nations.

Field, A. (2013). Discovering statistics using IBM SPSS statistics (4th ed.). Thousand Oaks, CA: Sage.

Goecker, A. D., Smith, P. G., Smith, E., \& Goetz, R. (2010). Employment opportunities for college graduates in food, renewable energy, and the environment: United States, 20102015. Retrieved from http://www.ag.purdue.edu/USDA/ employment/Documents/USDAEmployOp2010.pdf

Goecker, A. D., Smith, E., Marcos Fernandez, J., Ali, R., \& Goetz, R. (2015). Employment opportunities for college graduates in food, renewable energy, and the environment: United States, 2015-2020. Retrieved from http://www.ag.purdue.edu/USDA/employment/Documents/ USDAEmployOp2010.pdf

Herren, C. D. (2005). Perceptions of influences on college choice by students enrolled in the College of Agricultural Sciences and Natural Resources at Oklahoma State University (Masters thesis). Stillwater, OK: Oklahoma State University.

Hossler, D., Braxton, J., \& Coopersmith, G. (1989). Understanding student college choice. In J. Smart (Ed.). Higher education: Handbook of theory and research. New York: Agathon.

Hossler, D., \& Gallagher, K. S. (1987). Studying student college choice: A three-phase model and the implications for policymakers. College and University, 62(3), 207-221. Retrieved from http://www.aacrao.org/publications/candu/index.cfm

Howell, C. G. (2018). Social media marketing and college choice: A phenomenological study (Doctoral dissertation). Minneapolis, MN: North Central University.

Koon, L. A. F., Frick, M. J., \& Igo, C. G. (2009). What kind of students are enrolling in a college of agriculture and are they staying? A mixed methods approach. NACTA Journal, 53(2), 21-28. Retrieved from 
https://www.nactateachers.org/attachments/article/159/Koon_June_2009_NACTA_JOU NAL_final-4.pdf

Lingenfelter, K. M., \& Beierlein, J. G. (2006). Recruitment into the college of agricultural sciences: factors related to student major choices. Proceedings from the 2006 American Association of Agricultural Education National Research Conference, Charlotte, NC. Retrieved from http://aaaeonline.org/

Nora, A., Barlow, E., \& Crisp, G. (2005). Student persistence and degree attainment beyond the first year in college. College student retention: Formula for success. Washington, DC: American Council on Education.

Rayfield, J., Murphrey, T. P., Skaggs, C., and Shafer, J. (2013) Factors that influence student decisions to enroll in a college of agriculture and life sciences. NACTA Journal 57(1), 8893. Retrieved from https://www.nactateachers.org/attachments/article/2033/15\%20Rayfield\%20March\%202 013.pdf

Rocca, S. J. (2013). Comparison of factors influencing the college choice of matriculant and nonmatriculant students into a college of agriculture. NACTA Journal, 57(2), 72-78.

Retrieved from https://www.nactateachers.org/attachments/article/2068/13\%20Rocca\%20June13.pdf

Rocca, S. J., \& Washburn, S. G. (2005). Factors influencing the college choices of high school and transfer matriculants into a college of agriculture. NACTA Journal, 49(1), 32-38. Retrieved from https://www.nactateachers.org/attachments/article/353/Rocca_March_2005_NACTA_Jou rnal-6.pdf

Seidman, A. (2005). Minority student retention: Resources for practitioners. Washington, DC: New Directions for Institutional Research.

Shrestha, K. M., Suvedi, M., \& Foster, E. F. (2011). Who enrolls in agriculture and natural resources majors: A case from Michigan State University. NACTA Journal, 55(3), 33. Retrieved from https://www.nactateachers.org/attachments/article/1677/10_Shrestha_Dec2011.pdf

Smith, R. D. (2002). Strategic planning for public relations. Mahwah: Lawrence Erlbaum Associates, Inc.

Stair, K., Danjean, S., Blackburn, J. J., \& Bunch, J. C. (2016). A major decision: Identifying factors that influence agriculture students' choice of academic major. Journal of Human Sciences and Extension, 4(2), 111-125. Retrieved from https://docs.wixstatic.com/ugd/c8fe6e_f9d989c03a2c4638b7d3bafd66bed030.pdf

Tinto, V. (1993). Building community. Liberal Education, 79(4), 16-21. Retrieved from https://eric.ed.gov/?id=EJ479696

United Nations. (2015). World population prospects: The 2015 revision, (Data Booklet. ST/ESA/SER.A377). Retrieved from http://esa.un.org/unpd/wpp/Publications/Files/WPP2015_DataBooklet.pdf

Washburn, S. G. (2002). Factors influencing college choice for matriculants and nonmatriculants into a college of agriculture (Doctoral dissertation). Columbia, MO University of Missouri.

Westbrook, J. R., \& Alston, A. J. (2007). Recruitment and retention strategies utilized by 1890 land grant institutions in relation to African American students. Journal of Agricultural Education, 48(3), 123-134. https://doi.org/10.532/jae.2007.03123

Wildman, M., \& Torres, R. M. (2001). Factors identified when selecting a major in agriculture. Journal of Agricultural Education, 42(2), 46-55. https://doi.org/10.5032/jae.2001.02046 
Wildman, M. (1997). Factors in high school that influence choice of major by NMSU agriculture students (Masters thesis). Las Cruces, NM: New Mexico State University.

Williams, K.B. (2007). Factors influencing choice of academic major: A comparison of agricultural and non-agricultural degree programs (Master's thesis). Lubbock, TX: Texas Tech University.

Williams, K. \& Warren, J. (2014). Factors that influence a student to enroll in an agricultural science program at a selected university. International Journal of Science, Commerce, and Humanities, 2(6), 169-179. Retrieved from https://journals.indexcopernicus.com/search/details?id=35092 